\title{
Two-Step Resource Block Allocation Algorithm for Data Rate Maximization in LTE Downlink Systems*
}

\author{
Baorong Jiang ${ }^{1}$, Li Sun ${ }^{1,2}$, Pinyi Ren ${ }^{1}$ \\ ${ }^{1}$ School of Electronic and Information Engineering, Xi'an Jiaotong University, China \\ ${ }^{2}$ National Mobile Communications Research Laboratory, Southeast University, China \\ Email: jbr123@stu.xjtu.edu.cn, lisun@mail.xjtu.edu.cn, pyren@mail.xjtu.edu.cn
}

Received June, 2013

\begin{abstract}
This study focuses on resource block allocation issue in the downlink transmission systems of the Long Term Evolution (LTE). In existing LTE standards, all Allocation Units (AUs) allocated to any user must adopt the same Modulation and Coding Scheme (MCS), which is determined by the AU with the worst channel condition. Despite its simplicity, this strategy incurs significant performance degradation since the achievable system throughput is limited by the AUs having the worst channel quality. To address this issue, a two-step resource block allocation algorithm is proposed in this paper. The algorithm first allocates AUs to each user according to the users' priorities and the number of their required AUs. Then, a re-allocation mechanism is introduced. Specifically, for any given user, the AUs with the worst channel condition are removed. In this manner, the users may adopt a higher MCS level, and the achievable data rate can be increased. Finally, all the unallocated AUs are assigned among users without changing the chosen MCSs, and the total throughput of the system is further enhanced. Simulation results show that thanks to the proposed algorithm, the system gains higher throughput without adding too many complexities.
\end{abstract}

Keywords: Resource Block Allocation; Allocation Unit (AU); Two step; Re-allocation Mechanism

\section{Introduction}

Compared with 2G and 3G networks, The LTE systems can provide higher data rates and better transmission quality. Given limited radio resources, one challenge in LTE systems is to support a large number of users and satisfy different users' data rate requirements. Orthogonal Frequency Division Multiplexing (OFDM) is used as the basic transmission scheme in LTE downlink systems, which can achieve high system capacity. By using OFDM technique multiple users can share OFDM sub-carriers in a certain time slot [1-4]. In LTE systems, sub-carriers are grouped into Resource Blocks (RBs), and each RB consists of 12 adjacent sub-carriers. Two consecutive RBs are called an Allocation Unit (AU) [5]. In practical systems, all AUs allocated to a given user should use the same MCS, which is determined by the AU with the worst channel condition for this user.

Resource block allocation has been extensively studied in LTE systems. Proportional Fairness (PF) resource al-

The research reported in this paper was supported by the National Natural Science Foundation of China (No. 61201207), the National Science and Technology Major Project of China (No. 2012ZX03001005-004), the Open Research Fund of National Mobile Communications Research Laboratory, Southeast University (No. 2012D04), and the Fundamental Research Funds for the Central Universities of China. location mechanisms were presented in [5] and [6], which in reference [5] is composed of a priority adjustment scheme and a resource block allocation scheme. The priority adjustment scheme determines users' priorities according to channel conditions and average bit rate. The resource block allocation scheme allocates resource block to the user by PF mechanism, so the user's fairness is guaranteed. However, the users' data rate requirements are not taken into consideration in this allocation scheme. Resource block allocation scheme for data rate maximization is proposed in [7-9], the algorithm in reference [7] allocates AUs to the users according to their priorities determined by users' average channel conditions. In this manner, the minimum rate requirement of each user is satisfied. Nevertheless, for any user, the throughput is limited by the worst channel condition, and the AUs with better channel condition cannot be made full use of. In addition, if the users' minimum data rate requirements are too high, the users with lower priorities will not meet their minimum data rate requirements. It is not reasonable that the remaining AUs are just assigned to the user with the highest priority. In order to solve those problems presented above, a two-step resource block allocation algorithm is proposed in this paper.

The proposed algorithm consists of initial allocation 
scheme and re-allocation scheme. The initial allocation scheme allocates AUs to each user based on the number of AUs required by each user and its priority. The number of required AUs and the user's priority are determined by proportional fairness scheme. Next, for a certain user, the re-allocation scheme removes the AUs with the worst channel condition if the user's data rate is increased by doing so. Those removed AUs are saved as free AUs to re-allocate to the other users. If there are some AUs remained after each user has reached its minimum data rate requirement, the remaining $\mathrm{AU}$ is assigned to the user whose MCS is not higher than those of AU. As a result, the capacity of the proposed allocation algorithm has a better performance than the Qos algorithm and PF algorithm, while its complexity has not promoted so much.

The rest of this paper is organized as follows. Section 2 introduces the system model and presents the optimization objective function of resource block allocation for LTE systems. In section 3, the proposed resource block allocation algorithm is described. The simulation results are shown in Section 4. Finally, Section 5 makes a conclusion of this paper.

\section{System Model}

In LTE downlink transmission systems, we assume that $K$ active mobile users are distributed in a cell, and the total number of AUs is denoted by $N$. The considered system is shown in Figure 1. There are five users in one cell. Vector $\boldsymbol{c}_{k}=\left[c_{k, 1}, c_{k, 1}, \cdots c_{k, N}\right]^{T}$ indicates the Channel Quality Indicator (CQI) of user $\mathrm{k}$ for $\mathrm{N}$ AUs. The boxes at the top of figure represent the total AUs in LTE systems. The eNB allocates AUs to each user according to the feedback of CQI. The numbers in the boxes are the MCS indexes of corresponding AUs. We can see the AUs allocated to the same user have different MCS indexes, and the MCS of the user is determined by the AU with worst channel condition [10].

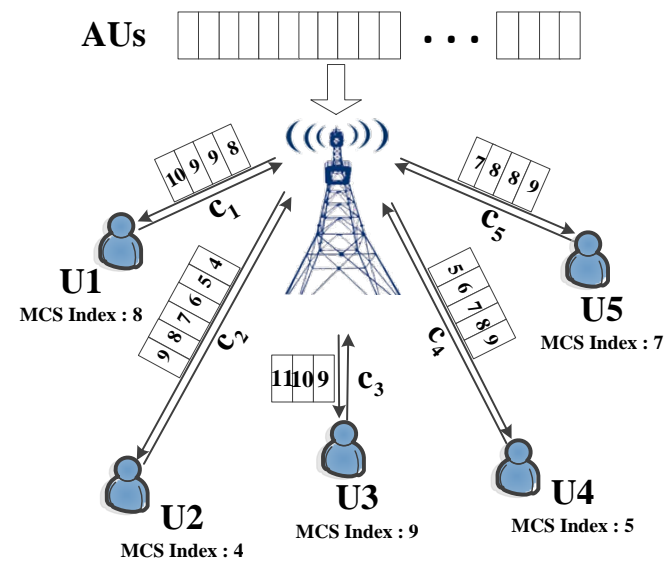

Figure 1. System model of resource block allocation in LTE downlink systems.
Let $c_{k, n}$ denote the CQI of user $k$ on AU $n$. The minimum data transmission rate of the user $k$ is indicated by $R_{k} \cdot r^{j}$ is the symbol transmission rate for a given Modulation and Coding Scheme (MCS) $j$, and $J$ is the total number of MCS supported in the transmission [11]. Define $N_{a u}$ as the number of symbols for data transmission in one AU. Set $T_{s}$ as the OFDM symbol duration time. Then, the bit rate transmitted in one AU can be calculated by:

$$
r_{a u}^{j}=\frac{r^{j} N_{a u}}{T_{s}}
$$

Variable $a_{k, n}$ represents the resource allocation indicator, with value 1 if AUn is allocated to user $k$ and 0 otherwise. Furthermore, define the variable $b_{k, j}$ as the choice of MCS of user $k$, and $b_{k, j}=1$ indicates that the MCS $j$ is chosen for user $k$. The data rate achieved by user $k$ can be described as:

$$
r_{k}=\sum_{n=1}^{N} a_{k, n} \sum_{j=1}^{J} b_{k, j} r_{a u}^{j}
$$

As the resource block allocation in this paper aims to achieve maximum sum data rate, the optimization problem is formulated as follows:

$$
\max _{a_{k, n}, b_{k, j}} \sum_{k=1}^{K} \sum_{n=1}^{N} a_{k, n} \sum_{j=1}^{J} b_{k, j} r_{a u}^{j}
$$

Subject to:

$$
\begin{gathered}
r_{k} \geq R_{k}, \quad \forall k \\
\sum_{k=1}^{K} a_{k, n}=1, \forall \mathrm{n}, \quad a_{k, n} \in\{0,1\} \\
\sum_{j=1}^{J} b_{k, j}=1, \quad \forall k, \mathrm{~b}_{k, j} \in\{0,1\}
\end{gathered}
$$

Constraint (3) is the objective function stating the achievable total rate, (4) ensures that each user can satisfy its minimum data rate requirement, (5) means that one AU can only be allocated to one user, (6) presents the fact that each user must use the same MCS, which is determined by the AU with the worst channel condition. It should be pointed out that finding the optimal solution would face high computational cost, so the suboptimal algorithm with low complexity is preferable

\section{Proposed Algorithm}

The algorithm in this paper is proposed based the allocation regulations of LTE downlink transmission. As user's MCS is determined by the AU with the worst channel condition, the data rate of each user is limited, and the AUs with the better channel condition are wasted. Therefore, a two-step resource block allocation algorithm focus on those problems is proposed in this paper. The 
first step of proposed algorithm initially allocates resource block by PF mechanism. The next step re-allocates the AUs with the worst channel condition of each user.

\subsection{Initial Allocation Scheme}

1) Estimating the number of required AUs:

In this paper, we calculate the number of AUs required based on the ratio of user's minimum data rate requirement to channel condition [4]. Let $\bar{c}_{k}$ be the average CQI of user $k . N_{k}$ is defined as the number of AUs allocated to user $k . N_{k}$ should satisfy the following conditions:

$$
\frac{R_{1}}{\overline{c_{1}}}: \frac{R_{2}}{\bar{c}_{2}}: \ldots: \frac{R_{k}}{\bar{c}_{k}}=N_{1}: N_{2}: \ldots: N_{k}
$$

which means the number of AUs each user needs is proportional to its minimum data rate requirement, and inversely proportional to the average channel condition. The number of AUs allocated to user $k$ is calculated by:

$$
N_{k}=\left\lfloor\frac{R_{k} / \bar{c}_{k}}{\sum_{k=1}^{K} R_{k} / \bar{c}_{k}} N\right\rfloor
$$

2) Calculating the priority of each user:

Reference [12] represents two methods to calculate the priority. The one is stated in Eq. (9), which means that the users with lower minimum rate requirements have higher priorities. The next one is formulated in Eq. (10), which indicates that the user with better channel condition could be first allocated. In this paper, we adopt a new way to calculate user's priority based on the variance of channel conditions and minimum data rate requirements simultaneously. The new method is given by Eq. (11), which implies that the user with better channel condition and lower data rate requirement has higher priority.

The first one:

$$
p_{k}=1 / \mathrm{R}_{k}
$$

The second one:

$$
p_{k}=\bar{c}_{k}=\frac{1}{c_{k}} \sum_{n=1}^{N} c_{k, n}
$$

The third one:

$$
p_{k}=\sigma_{k}^{2} / \mathrm{R}_{k}, \sigma_{k}^{2}=\sum_{n=1}^{N}\left(c_{k, n}-\bar{c}_{k}\right)^{2}
$$

3) Allocating AUs to each user

The initial allocation scheme is described in Table $\mathbf{1}$, which allocates $N_{k}$ AUs to the user $k$ according to the users' priorities and the number of AUs each user needs.
Meanwhile, the minimum data rate requirement of each user is guaranteed.

\subsection{Re-allocation Scheme}

In LTE systems, if AUs allocated to the same user have different channel qualities in terms of CQI, the MCS is determined by the lowest CQI to make sure that the data transmitted by each AU can be correctly received. Obviously, the users' data rate is limited by the AUs with the worst channel condition, and the AUs with better channel condition cannot be made full use of. A solution for above problem is proposed, which is explained in Figure 2. The number of AUs allocated to each user in Figure 2 is the same as Figure 1. Assuming that each AU only consists of one symbol, the symbol transmission rate can be found in Table 2. 'Before' in Figure 2 is MCS index and data rate obtained by the user without removing any AUs. 'DR' in Figure 2 is abbreviation of data rate. Take user 2 for example, if the AU with the worst channel condition is removed, MCS index of this user turns into 5, and the data rate of this user is increased. The dotted boxes indicate the removed AUs, Which is saved as free AUs to allocate to other users, such as the first AU adopted to user 3 is removed by user 2, and the MCS indexes of AUs allocated to user 3 turn into higher level.

Table 1. The Initial Allocation Scheme.

\begin{tabular}{l} 
Initial Allocation \\
\hline $01 . \quad$ Estimate the number of AUs required by according to \\
the Eqs. (8) and (9); \\
$02 . \quad$ Calculate users' priorities based on the Eqs. (10), (11) \\
or (12), and sort them in descending order; \\
03. Find out the first $N_{k}$ AUs with better channel condition \\
from available AUs for user $k$; \\
$04 . \quad$ Calculate the data rate of user $k$ by Eq. (1), if it is less \\
than its minimum data rate requirement, allocate one more \\
AU to this user until the minimum requirement is met.
\end{tabular}

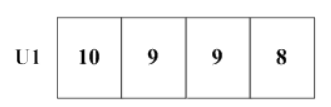

$$
\begin{aligned}
& \text { MCS : } 8 \\
& \text { DR : } 7.6564
\end{aligned}
$$
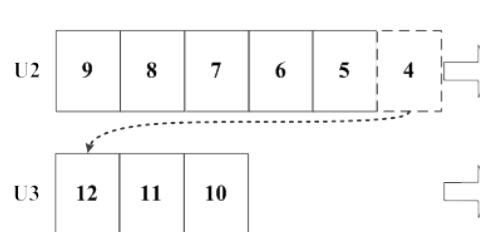

Before:

MCS: 4, DR: 3.6096 After: MCS: 5, DR: 4.3850 Before:
MCS: 9, DR: 7.2189 After:

MCS: 10, DR:8.1915

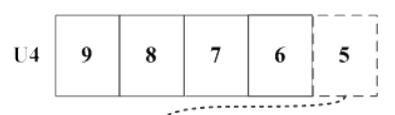
Before:
MCS: 5, DR: 4.3850
After:
MCS: 6, DR: 4.7032

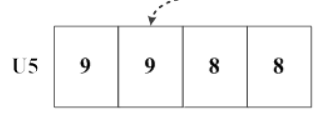
Before: Befe: 7 , DR: 5.9064
Mfter:
MCS: 8, DR: 7.6564 
Figure 2. The example to illustrate proposed scheme.

Moreover, there are several AUs remaining in practical applications after users have reached their minimum data rate requirements. Those remaining AUs should be assigned without changing the MCS of each user, that is to say, we allocate the remaining AUs to users, whose MCS are not higher than those of remaining AU. By this way, the total number of AUs allocated to some users is added, so the data rates achieved by these users are improved. The re-allocation scheme is stated in Table 2.

\section{Simulation Results}

This section compares the performance of the proposed scheme, Qos algorithm [7], and PF algorithm [5] and max-rate algorithm. The simulation parameters are shown in Table 4. All simulations are obtained by averaging over 1000 channel realizations [13-14].

Table 2. MCS Table in LTE Systems.

\begin{tabular}{cccccc}
\hline MCS Index & 1 & 2 & 3 & 4 & 5 \\
\hline$r$ (bit/symbol) & 0.1523 & 0.2344 & 0.3770 & 0.6010 & 0.8770 \\
MCS Index & 6 & 7 & 8 & 9 & 10 \\
$r$ (bit/symbol) & 1.1758 & 1.4766 & 1.9141 & 2.4063 & 2.7305 \\
MCS Index & 11 & 12 & 13 & 14 & 15 \\
$r$ (bit/symbol) & 3.3223 & 3.9023 & 4.5234 & 5.1152 & 5.5547 \\
\hline
\end{tabular}

Table 3. The Re-allocation Scheme.

\begin{tabular}{ll}
\hline & \multicolumn{1}{c}{ Re-allocation scheme } \\
\hline 01. & For $k=1: K$ \\
02. & Allocate $N_{k}$ AUs to user $k$, and calculate $r_{k}$ of this user; \\
03. & While $r_{k}<R_{k}$ \\
04. & allocate one more AU to the user; \\
05. & End While \\
06. & Calculate data rate $r_{k}$ without allocation of AUs with \\
07. & the worst channel condition; \\
08. & If $r_{k} \geq r_{k}$ \\
09. & Remove AUs with the worst channel condition. \\
10. & Then $k=k+1$, go to step 1 ; \\
11. End If \\
12. End For \\
13. $\quad$ Allocate the remaining AUs to users, whose MCS are not \\
higher than those of remaining AU.
\end{tabular}

Table 4. Simulation Parameters.

\begin{tabular}{cc}
\hline Simulation Parameters & Values \\
\hline System bandwidth & $20 \mathrm{MHZ}$ \\
Carrier frequency & $2 \mathrm{GHZ}$ \\
Sampling frequency & $30.72 \mathrm{MHZ}$ \\
Channel model & $\mathrm{SCM}$ \\
Number of AUs & 50 \\
Number of users & $6-18$ \\
Channel feedback & Full \\
OFDM symbols per frame & 14 \\
TTI duration & $1 \mathrm{~ms}$ \\
& QPSK:1/3,1/2,2/3,3/4,4/5 \\
\hline
\end{tabular}

\begin{tabular}{cc}
\hline Simulation Parameters & Values \\
\hline Modulation and coding & 16QAM:1/2,2/3,3/4,4/5 \\
& 64QAM:2/3,3/4,4/5 \\
\hline
\end{tabular}

To illustrate user's fairness achieved by each algorithm, the fairness factor is introduced [2], which is stated as:

$$
I=\left(\sum_{k=1}^{K} T_{k}\right)^{2} / K \sum_{k=1}^{K} T_{k}^{2}
$$

where $I$ is the fairness factor. $T_{k}$ is the average data rate of user $k$. The closer the fairness factor obtained by algorithm approximates to 1 , the better the fairness of the algorithm is.

Figure 3 compares fairness index of the proposed scheme, Qos algorithm, PF algorithm and max-rate algorithm. As the max-rate algorithm aims to maximize the system throughput without considering users' requirements, it displays the worst in terms of fairness. With the increasing of the number of users, the fairness factor of max-rate algorithm is decreased sharply. The fairness factors of the other three algorithms are close. Their fairness factors are all more than 0.9 when the number of users is less than 14. The proposed algorithm exhibits the best fairness in these three algorithms, and Qos and PF algorithm are slightly lower than it.

The throughput of the system is shown in Figure 4. Because the users' requirements and remaining AUs are not considered, the throughput of PF algorithm is the lowest of all. From the point of throughput, the proposed algorithm is superior to the Qos and PF algorithm, benefiting from higher MCS of some users, free AUs, and the appropriate allocation of remaining AUs. Max-rate algorithm displays the best performance of the four algorithms. The curve of proposed algorithm is close to that of the max-rate algorithm. When the number of users is small, the curve of proposed algorithm is higher than that of max-rate algorithm. Because of the MCS selection mechanism in LTE systems, max-rate algorithm cannot always provide the best performance. 


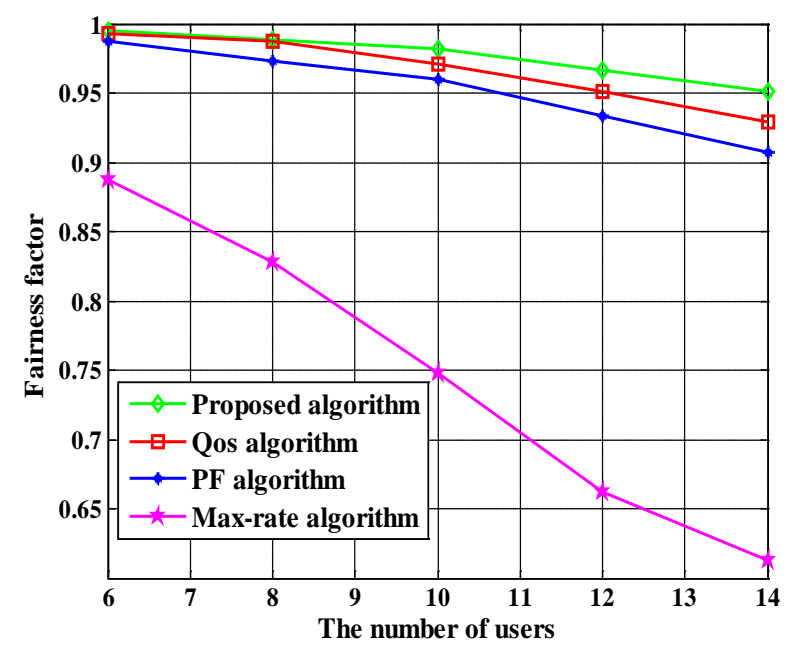

Figure 3. The fairness factors of four algorithms.

The unreached rate is used to illustrate the Qos guaranteed of each algorithm. The smaller the unreached rates gained by the algorithm, the better the user's Qos guaranteed is. The unreached rate can be calculated by:

$$
r_{u}=\sum_{k=1}^{K} P_{k}, \quad P_{k}=\left\{\begin{array}{cc}
R_{k}-r_{k}, & R_{k}>r_{k} \\
0, & \text { otherwise }
\end{array}\right.
$$

where $r_{u}$ is the total unreached rate in the system. $r_{k}$ is the data rate achieved by user $k . P_{k}$ is the unreached rate of user $k$.

Figure 5 demonstrates the unreached rate of the users among the proposed algorithm, Qos algorithm, and PF algorithm. The PF algorithm allocates AUs without considering the requirements of users, so unreached rates gained by $\mathrm{PF}$ algorithm rapidly increase as the number of users increases. It can be found that Qos algorithm guarantees users' requirements well, and the unreached rate achieved by Qos algorithm is not too high. The proposed algorithm keeps the unreached rate at the lowest level,

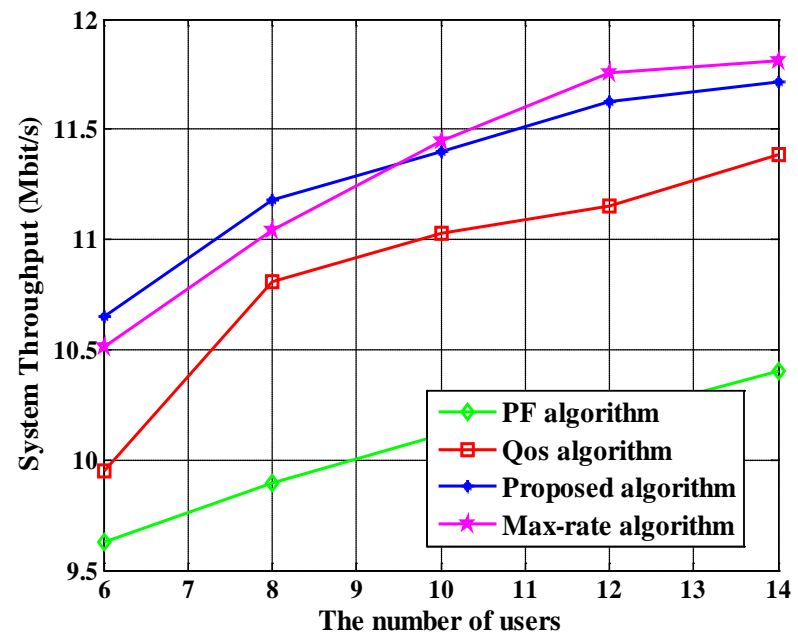

Figure 4. System throughput of four algorithms.

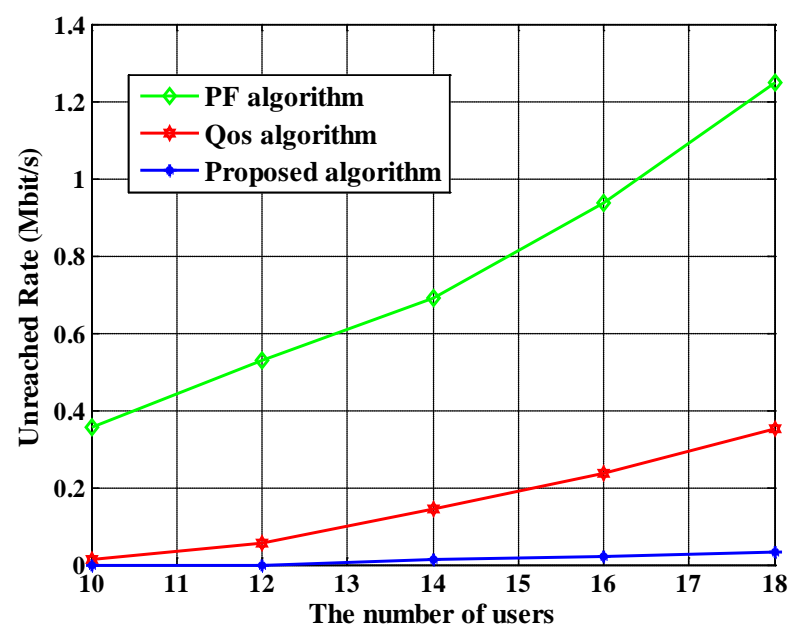

Figure 5. The unreached rate of three algorithms.

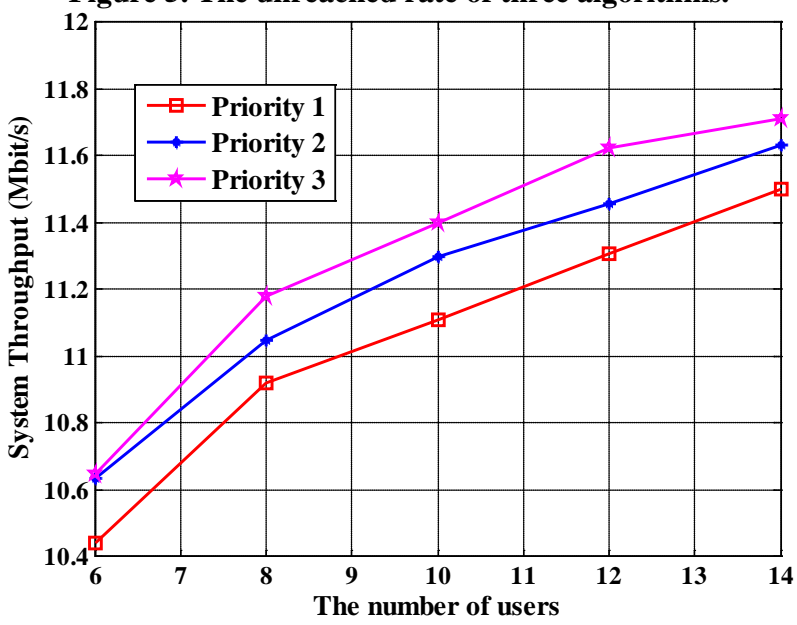

Figure 6. System throughput of proposed algorithm with different methods for determining the priorities.

Table 5. Computational complexity of four algorithms.

\begin{tabular}{lcccc}
\hline \multicolumn{1}{c}{ Algorithms } & Max-rate & PF & Qos & Proposed \\
\hline Initial allocation & $O(K N)$ & $O\left(K N^{2}\right)$ & $O\left(K N^{2}\right)$ & $O\left(K N^{2}\right)$ \\
Qos guarantee & $\backslash$ & $\backslash$ & $O(K N)$ & $O(K N)$ \\
Re-allocation & $\backslash$ & $\backslash$ & $\backslash$ & $O(K N)$ \\
Total complexity & $O(K N)$ & $O\left(K N^{2}\right)$ & $O\left(K N^{2}\right)$ & $O\left(K N^{2}\right)$ \\
\hline
\end{tabular}

close to zero. The unreached rate attained by proposed algorithm is not more than $0.1 \mathrm{Mbit} / \mathrm{s}$ when the number of users is less than 18.

Figure 6 shows the throughput of proposed algorithm with different methods for determining priorities. Obviously, the priority considered users' requirements and channel conditions simultaneously presents the best performance of all. The performance gained by the priority determined by the channel condition is superior to that gained by the priority determined by minimum data rate requirement. 
Table 5 shows the computational complexity of the four algorithms. We can easily learn that the complexity of max-rate algorithm is $O(K N)$, and the complexity of $\mathrm{PF}$ and Qos algorithm are all $O\left(K N^{2}\right)$. For the proposed algorithm, the complexity of initial allocation is $O\left(K N^{2}\right)$, and the complexity of re-allocation scheme is $O(K N)$, Therefore, the overall complexity of proposed algorithm is $O\left(K N^{2}\right)+O(K N)=O\left(K N^{2}\right)$. It is observed that, the proposed algorithm gains the quadratic complexity, which is the same as that of PF and Qos algorithm. The last column of Table 5 explains that the max-rate algorithm gets the lowest computational complexity, and the other three algorithms have the same computational complexity.

\section{Conclusions}

In this paper, we propose a two-step resource block allocation algorithm for LTE downlink systems. The first step of proposed algorithm initially allocates AUs to each user based on the proportional fairness scheme. Then, the proposed scheme re-allocates the AUs with the worst channel condition of each user to improve the performance restriction by those AUs. The algorithm can provide us with a higher data rate and a flexible allocation scheme, and ensures the user's fairness. Simulation results show that the proposed algorithm provides higher throughput and ensures the fairness of each user.

\section{REFERENCES}

[1] G. Ioannis, Fraimis and S. A. Kotsopoulos, "QoS-Based Proportional Fair Allocation Algorithm for OFDMA Wireless Cellular Systems," IEEE Communications Letters, Vol. 15, No.10, 2011, pp. 1091-1093.

doi:10.1109/LCOMM.2011.081211.111417

[2] C. F. Tsai, C. J. Chang, F. C. Ren and C.-M. Yen, “Adaptive Radio Resource Allocation for Downlink OFDMA/SDMA Systems with Multimedia Traffic," IEEE Trans. Wireless Commum., Vol. 8, No. 5, 2009, pp. 2927-2937.

[3] J. C. Fan, Q. Y. Yin, G. Li and B. G. Peng, "Adaptive Block-Level Resource Allocation in OFDMA Networks," IEEE Transactions on Wireless Communications, Vol. 10, No. 11, 2011. pp. 3966-3972. doi:10.1109/TWC.2011.092011.110624
[4] Leinonen, Jouko, Hamalainen, Juntti and J. Markku, "Performance Analysis of Downlink OFDMA Resource Allocation with Limited Feedback," IEEE Transactions on Wireless Communications, Vol. 8, No. 6, 2009, pp. 2927-2937. doi:10.1109/TWC.2009.071374

[5] R. Kwan, C. Leung and J. Zhang, "Proportional fair multiuser scheduling in LTE,” IEEE Signal Processing Letters, Vol. 16, No. 6, 2009, pp. 461-464. doi:10.1109/LSP.2009.2016449

[6] I-Hong Hou, Chungshu Chen, "Self-Organized Resource Allocation in LTE Systems with Weighted Proportional Fairness," Proceedings of the IEEE International Conference on Communications, Ottawa, June 2012, pp. 5348-5354.

[7] Y. Q. Zhou, L. Tian, G. Sun and J. L. Shi, “QoS guaranteed resource block allocation algorithm for LTE systems," Proceedings of the IEEE 7th International Conference on WiMob, Wuhan, Oct. 2011, pp. 307-312.

[8] R. Kwan, C. Leung and J. Zhang, "Resource allocation in an LTE cellular communication system," Proceedings of the IEEE International Conference on Communications, Dresden, June 2009, pp. 1-5.

[9] L. Q. Zhao, Y. Qin, M. Ma, X. X. Zhong and L. Li, “QoS Guaranteed Resource Block Allocation Algorithm in LTE Downlink," Proceedings of the 7th International ICST Conference on CHINACOM, Kun Ming, Aug. 2012, pp. 425-429.

[10] L. Sun, P. Wang and F. Q. Liu, "Particle Swarm Optimization based Resource Block Allocation Algorithm for Downlink LTE Systems," Proceedings of the 18th Asia-Pacific Conference on Communications, Jeju Island, Oct. 2012, pp. 970-974.

[11] J. C. Fan, Q. Y. Yin, G. Y. Li, B. G. Peng and X. L. Zhu, "MCS Selection for Throughput Improvement in Downlink LTE Systems," Proceedings of the 20th International Conference on ICCCN, Maui HI, Aug. 2011, pp. 1-5.

[12] C. C. Wen, C. J. Chang and L. C. Wang, "An Intelligent Priority Resource Allocation Scheme for LTE-A Downlink Systems,” IEEE Commun. Lett., Vol. 1, No. 3, 2012, pp. 241-244.

[13] 3GPP TS 36.211 v.9.1.0, Evolve Universal Terrestrial Ratio Access; Physical Channel and Modulation, May 2010.

[14] J. C Ikuno, M. Wrulich and M. Rupp, "System Level Simulation of LTE Network," Proceedings of the IEEE 71st Vehicular Technology Conference, Taipei, May 2010, pp. 1-5. 